\title{
scRNA-Seq Identifies IL-1 Responsive Cell Subsets in the Skin Injury-induced Inflammatory Response
}

Kayla Harpold ${ }^{1}$, Hong-Ming Zhou ${ }^{2}$, Radomir M. Slominski ${ }^{2}$, Leroy J. Seymour ${ }^{2}$, Maria C. Bell $^{1}$, Priya Dave ${ }^{8}$, Joseph Atumonye ${ }^{1}$, William Wright III ${ }^{8}$, Avery Dawes ${ }^{2}$, Brad Griesenauer $^{3,5,7}$, Sophie Paczesny ${ }^{3,5,7}$, Mark H. Kaplan ${ }^{3,5,6}$, Dan F Spandau ${ }^{2,6}$, Yunglong Liu $^{9,10,11,12}$, Xiaoling Xuei ${ }^{10}$, Hongyu Gao ${ }^{10}$, Aki Hoki ${ }^{4}$ \& Matthew J. Turner ${ }^{2,3,13}$

Indiana University School of Medicine ${ }^{1}$; Departments of Dermatology2; Microbiology \& Immunology3; Pediatrics ${ }^{4}$; Biochemistry \& Molecular Biology6; Melvin and Bren Simon Cancer Center ${ }^{7}$; Life-Health Science Internship Program, IUPUI ${ }^{8}$; Center for Computation Biology and Bioinformatics ${ }^{9}$; Medical \& Molecular Genetics ${ }^{10}$; Biostatistics ${ }^{11}$; Informatics and Computing ${ }^{12}$; Richard L. Roudebush VA Medical Center ${ }^{13}$

Inflammation is an integral aspect of skin wound healing; however, the mechanisms that regulate inflammatory cascades in this context are not well defined. To better understand how skin inflammation impacts wound healing, we developed an ex vivo skin culture system to model key aspects of the inflammatory phase of wound healing. In this model, a defined set of proinflammatory cytokines and chemokines, mirroring those produced in wounds in vivo, are produced when mouse or human skin biopsies are cultured ex vivo. We refer to this pattern of cytokine and chemokine induction as the skin injury-induced inflammatory response. Previous studies in our laboratory demonstrated this response is initiated by the cytokine, interleukin 1

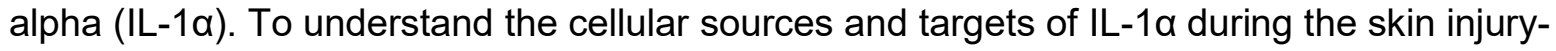
induced inflammatory response, skin biopsies from mouse tail skin were cultured ex vivo for 8 hours followed by processing for single cell RNA sequencing (scRNAseq). Using bioinformatic software, $\mathrm{R}$, and the package, Seurat, analysis of scRNAseq data from this experiment identified 22 distinct cell population clusters. While no populations exhibited significant expression of $/ / 1 \mathrm{a}$ transcripts, multiple cell populations expressed $/ / 1 r 1$ transcripts, which encodes the ligand-specific subunit of the IL-1 receptor. Notably, fibroblast, endothelial cell and stromal cell clusters were characterized by expression of $I / 1 r 1$ and the skin injury-induced inflammatory response transcripts //6, Cxc/1 and/or Csf3. Furthermore, Reactome Pathway Analysis suggested the II-1 signaling axis was activated in these cell populations. This information provides a basis for future studies to understand how IL-1 signaling in fibroblasts, endothelial cells and stromal cells impacts wound healing in vivo, which could in turn lead to novel therapeutic approaches to clinically relevant outcomes. 